\title{
Decades of water safety training culturally "irrelevant" to First Nation people
}

Published at www.cmaj.ca on July 26

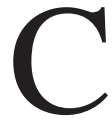

anada's Aboriginal people and others at high risk of drowning are among those least served by conventional water safety training, say injury prevention experts.

"There's been a longstanding stigma that Aboriginal people are too stupid or don't want to learn about water safety, and that's just ridiculous," says Audrey Giles, an associate professor in the School of Human Kinetics at the University of Ottawa in Ontario. "Aboriginal communities on frozen waterfronts get sent posters and instructions targeted at white children in heated swimming pools. We shouldn't be shocked that this isn't getting the right message across."

Some 205 people have drowned in Canada since January, according to a July tally by the Lifesaving Society, a national nonprofit organization that has been training lifeguards since 1908 . That's 25 more drowning deaths than were totalled at the same time last year. First Nation and Inuit peoples, particularly those living in the north, are among those at highest risk. The Lifesaving Society and the Canadian Red Cross estimate that Aboriginal drowning rates are 6-10 times higher than the national average.

The exact burden, however, is unclear, says Giles. "Ethnicity isn't tracked on coroner's reports, so the estimate is based on where the person drowned, if it was an Aboriginal community, and whether or not the person's name looks Aboriginal. There's a real need for someone to do an exhaustive review of the issue."

As evidence of the role culture plays in risk communication increases, experts say the lack of cultural awareness in the delivery of some drowning prevention programs poses a major obstacle to improving water safety.

"We take information developed in and for the south [of Canada], typically

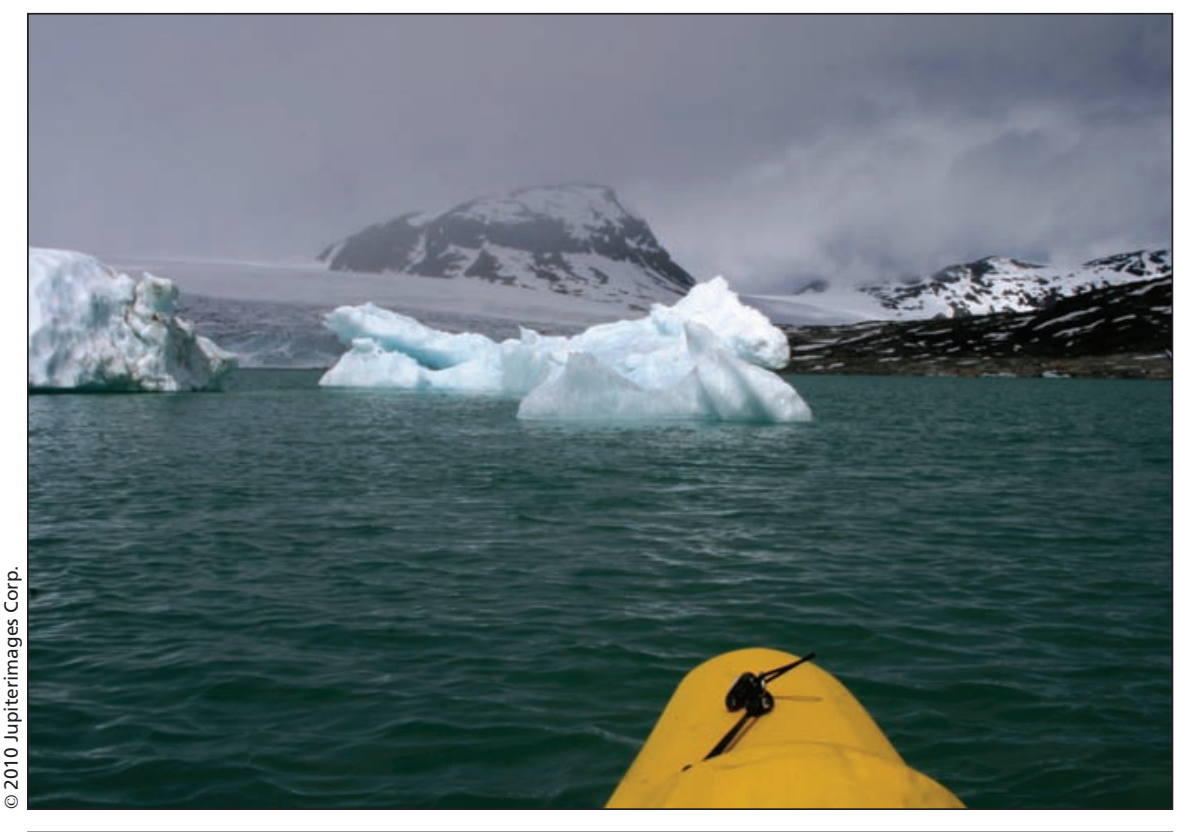

Conventional water safety programs designed for swimming pools fail to account for the harsh conditions and icy waters encountered by aboriginal communities on natural waterfronts.

by Euro-Canadians, and we export it and expect it to work in the rest of the country. For all the decades we've been delivering these programs, Aboriginal drowning rates are still way above the national average. But it's not surprising that northern communities are unwilling to implement strategies that haven't been developed for them or with their input," says Giles.

From 2006-2010, Giles toured Canada's north to research water safety practices and perceptions among First Nation and Inuit peoples. Over the course of 120 interviews, she found that many Aboriginal communities, despite expressing a need for drowning prevention programs, felt the current training available was "irrelevant" to life in the north. A capstone summary of Giles' findings will be published within the year.

Implementing swimming and water safety programs in Aboriginal communities has always been a challenge due to a dearth of aquatic facilities and lifesaving equipment, says Deanna JonesKeeshig, injury prevention coordinator for Chiefs of Ontario, a body that represents 134 First Nation communities. "Only a handful of these communities have recreation centres, let alone a pool. Not to mention the cost of shipping life jackets out to these communities is really prohibitive, especially to buy them for the whole family and replace them as kids grow."

Some communities can use pools in neighbouring towns, but even those, Jones-Keeshig says, are few and far between outside major cities. "The smaller municipalities are in the same boat we are."

Those who do have access to local pools for swimming lessons may not be prepared by standard training for the conditions they'll encounter in cold lakes and rivers, says Carl Shier, CEO of the Manitoba branch of the Lifesaving Society. 
"Aboriginal people are using boats and water taxis, they're down by the rocks at shore's edge, they're on docks and they're paddling in rivers. The conditions are never ideal like they are in swimming pools, and even for the best swimmers the body will perform differently in cold or freezing water."

Many of the summer instructors hired to teach swimming and survival courses to Aboriginal people are university students with little or no exposure to the culture, geography and basic conditions of life in the communities they visit, says Giles. "A 19- or 20year-old student from a city won't know where the water is dangerous, or where the ice is thin. There's no place in these programs for traditional knowledge, or for the participation of community elders who may not have formal certification but have years of experience on the land."

Not accounting for traditional knowledge can mean dangerous gaps in safety information, Giles adds. "In the north, the ice doesn't melt completely in the summer. If you have a knife with you when you go overboard, you can stab a chunk of floating ice and pull yourself out of the water. And having a rifle is essential when you're out on the land because you never know when you could be stranded by the weather and need to hunt for food. These are crucial survival tools, but you'll never see them listed on a standard predeparture checklist."

According to Jones-Keeshig, climate change has made local participation in water safety programs more necessary than ever. "Our people are saying that what we once knew as the landscape, has changed. You've got water in places you didn't before, creeks turning into rivers, and unmarked shallows. The only way you can know about these is from people reporting back from the land."

Programs that exclude or ignore existing practices in a community also run the risk of alienating participants, Giles says. "The Inuit have their own culturally-tied notions of what you should do for someone who has been submerged in water that look very different from CPR. If you don't recognize that when you're offering CPR training you can end up marginalizing that community unintentionally."

Cultural gaps in water safety training are also affecting other groups at high risk of drowning.

A recent survey by the Lifesaving Society found that new Canadians are almost five times less likely to know how to swim (www.lifesaving.bc .ca/download/NDPW/LSResearchBack grounder.pdf). Like Aboriginal communities, differences in language, culture and access to aquatic facilities can pose barriers to delivering safety strategies to recent immigrants, says Shier.

Adult males of all ethnic backgrounds are also at a high risk of drowning. The fact that most water safety programs are targeted at children and taught by teenagers often prevents adult males from taking risk prevention messages seriously, says Giles.

"There's an assumption that you learn to swim as a kid and the skill is with you for life, but considering the length of time water safety programs have been available in Canada, that's obviously not the case," she adds. "We think children are the only ones that are vulnerable, but, in fact, we do a better job protecting them than what most adults do of protecting themselves."

In the last five years, some organizations have begun to implement changes to provide more culturally relevant water training.

Manitoba's Lifesaving Society now runs a water survival program tailored to the north, and uses a video in training that stars Aboriginal people sharing safety stories. They've also made changes to instructor presentations on life jackets.

"We've taught our instructors to emphasize that, while a life jacket may not save its wearer's life, it will increase their chances of being found. The reality is these communities will put everything on hold until a missing person is found, and most of the adults have been on recovery missions at one time or another in the worst possible weather conditions," says Shier. "Knowing that wearing a life jacket will make that search safer for the community is a higher priority than whether or not it saves your life, and that's something people outside these communities didn't get in the past."

Three years ago, the Manitoba branch of the society started a loaner program to distribute personal flotation devices to Aboriginal communities. They also deliver water safety presentations to English classes for recent immigrants. Earlier this year, the Ontario branch of the society translated boating safety materials into 33 languages, including Cree, Ojibway, Ojicree and Mohawk.

Also this year, the Canadian Red Cross finished a pilot project in partnership with Aboriginal communities in Ontario to develop and deliver swimming lessons and water safety instructor training.

Like the Lifesaving Society, the Red Cross learned to incorporate traditional storytelling techniques into their programming, along with expanded safety tips to cover common activities in Aboriginal communities, such as driving all-terrain vehicles near water and on the ice.

Since the development of these programs, Manitoba has seen a decrease in Aboriginal drowning rates. In 1991, the province had the highest number of Aboriginal water-related deaths nationwide, according to a Lifesaving Society tally from coroners' data. Now, Aboriginal drowning rates are down by about $25 \%$, says Shier.

It's difficult to track the success of these programs because there's no national strategy on drowning prevention for Aboriginals, says JonesKeeshig. Organizations have to rely on coroners' data and media reports to estimate the number of water-related deaths each year.

Chiefs of Ontario will release an environmental scan on injuries and injury prevention in the province's First Nation communities in the fall. Lauren Vogel, CMAJ

DOI:10.1503/cmaj.109-3323 